\title{
LA GESTIÓN DE DOCUMENTOS EN LAS INSTITUCIONES DE EDUCACIÓN SUPERIOR: DE UN PROCESO OPERATIVO A UN PROCESO ESTRATÉGICO
}

\author{
Raquel Umaña Alpízar*
}

\section{Resumen}

Este artículo presenta la historia y los avances en la aplicación de las buenas prácticas de gestión documental en las instituciones de educación superior en Costa Rica, producto de los cambios en la gestión universitaria por la implantación de la administración electrónica, como componente fundamental para la correcta implantación de la UDigital.

Palabras clave: gestión de documentos, archivos universitarios, normalización, proceso estratégico.

\section{Summary}

This article shows the history and progress on the implementation of document management's good practices on institutions of higher education in Costa Rica, due to changes in university's management by the implementation of e-government, as essential component for successful implementation of UDigital program.

Keywords: records management, university archives, standardization, strategic process.

* Universidad de Costa Rica. 


\section{Introducción}

El auge de las tecnologías en el mundo actual ha provocado un flujo constante cuyos efectos -en mayor o menor grado- se revelan en las organizaciones. Nadie escapa a las consecuencias del avance tecnológico; incluso las organizaciones tienen que enfrentar y adaptarse a esta transformación - producto de la evolución o, más bien, de la revolución de las tecnologías y su indeleble impacto en la generación y la comunicación del conocimiento-, que obliga a las instituciones a adaptarse a los requerimientos de la sociedad.

Las llamadas "nuevas tecnologías de la información y comunicación" eliminan los impedimentos de distancia y tiempo, contribuyen al crecimiento, la transferencia y la multiplicación de la capacidad de almacenamiento de la información. Como producto de estas innovaciones, el valor estratégico de la información y su aplicación práctica es abordado por teóricos de diferentes disciplinas y campos de la actividad humana.

De acuerdo con lo anterior, la sociedad se transforma aceleradamente; las instituciones intentan igualar tal ritmo con cambios -para mejorar y adecuar oportunamente las metas, los procesos y actividades-, mediante un sano equilibrio entre continuidad y cambio que les permite analizar y comprender sus procesos internos.

Tanto en el ámbito empresarial como estatal, la eficiencia de esos procesos -sean del sector producción o servicios-, la gestión de los recursos y la competitividad están fundamentados en el acceso, veraz, confiable y oportuno, de la información. Cada segmento de datos puede ser visto como bloques que generan ideas y cambios, o como insumos de valor sobre las tendencias y necesidades actuales y las que se proyectan para el futuro.

La gestión de documentos, relativa a conseguir economía y eficacia en la creación, control, mantenimiento, uso y acceso de los documentos es, entonces, garante de la información en custodia, de su disponibilidad presente y futura y de su acceso expedito y oportuno; por tanto, estas funciones y procesos dan respuesta a los desafíos del entorno digital propio de nuestros tiempos.

De acuerdo con lo anterior, la gestión documental es el soporte de todo sistema de calidad, pues en ella se plasman no sólo las formas de operar una organización, sino, la información que permite el desarrollo de sus procesos, la toma de decisiones, la comunicación, la transparencia y la rendición de cuentas. 
El presente trabajo expone la sistematización de un proceso que se alimenta de la construcción conjunta de un grupo de profesionales, quienes enfrentan el reto de la normalización y de transportar la práctica de la gestión documental del siglo XX al XXI.

\section{Los archivos universitarios}

Hace pocos años la realidad de los documentos en nuestras instituciones de educación superior era, por decir poco, desafortunada. Sin embargo, tras laboriosos esfuerzos de profesionales visionarios, las universidades estatales se han concientizado sobre la importancia de la organización de los documentos y su valor como elementos indispensables para su desempeño y desarrollo.

De entre las instituciones públicas de educación superior en Costa Rica, la que dio el primer paso en una dirección correcta fue el Instituto Tecnológico de Costa Rica (Tec), que mediante acuerdo del Consejo de Rectoría -tomado en la sesión No 5-77 del 9 de febrero de 1977-, aprueba la creación del Centro de Archivo y Correspondencia, hoy Centro de Archivo y Comunicaciones, el cual, desde su inicio, contó con un manual de clasificación a nivel institucional que permitió organizar en forma eficiente sus documentos.

Siguiendo el ejemplo, se crea el Archivo Central de la Universidad Estatal a Distancia (UNED), que inicia formalmente sus funciones a principios de 1986, adscrito al Centro de Información y Documentación Institucional (CIDI); en 2005 es reubicado jerárquicamente, ahora como dependencia de la Vicerrectoría Ejecutiva.

Aprovechando el auge que impulsó la creación del Sistema Nacional de Archivos -mediante la aprobación de la Ley No 7202, Ley del Sistema Nacional de Archivos, y su Reglamento- en 1990 se redefine el papel de los archivos de las instituciones públicas al incorporarlos como miembros activos de un sistema nacional.

El Archivo de la Universidad Nacional, fundado en 1993, es un ejemplo del nuevo paradigma. Este proyecto inició en 1987 como un Sistema Institucional de Archivos, pero no fue sino hasta 1992 -con la promulgación de la Ley 7202- que toma el impulso necesario para llegar al Consejo 
Universitario, preocupado por la organización de sus documentos. Por lo anterior, se nombra una comisión interdisciplinaria de Archivo central, que fue la instancia encargada de coordinar las acciones necesarias para lograr la implementación del Sistema de Archivo Universitario.

En la Universidad de Costa Rica (UCR) -la institución de educación más antigua-, a pesar de que el M. Sc. Eduardo Fournier García propone, en 1985, crear un Archivo Central, no es sino hasta 2003 que se crea el Archivo Universitario Rafael Obregón Loría (AUROL), con la misión de custodiar y salvaguardar el acervo documental de la institución mediante acciones de recuperación y conservación.

El 2 de septiembre de 2008 el Consejo Universitario -sesión 5282, artículo 5- aprueba el Reglamento del Sistema de Archivos Universitarios de la Universidad de Costa Rica y decreta que el Aurol será el encargado de organizar y dirigir el sistema de archivos con el fin de coordinar la función archivística universitaria, salvaguardar el patrimonio documental de la institución y facilitar el acceso a la comunidad.

Este reglamento fue analizado por una comisión interdisciplinaria que se originó a partir de la resolución del Sexto Congreso Universitario -titulada "El impacto tecnológico en la gestión documental en la administración universitaria"-, la cual, desde entonces, deja claro que la visión de sus precursores era actualizar la institución en materia de orientación de la gestión documental como instrumento para enfrentar el creciente desarrollo de las tecnologías de información y su uso expansivo en todas las áreas del quehacer de la comunidad universitaria.

La creación del AUROL se benefició con el apoyo de los encargados de los archivos de otras universidades públicas, quienes en varias reuniones -incluso mientras la Universidad de Costa Rica daba forma a su proyecto de archivo universitario- consideraron crear una instancia de coordinación bajo las directrices del Consejo Nacional de Rectores (Conare); como consecuencia, se forma en 1999 la Comisión de Archivos Universitarios (CAU-Conare), órgano colegiado que ha integrado representantes de varias instituciones para intercambiar experiencias y analizar los problemas archivísticos en las universidades, y al que, posteriormente, se suscribiría el nuevo archivo universitario -que ha pugnado por el mejoramiento continuo de los sistemas institucionales de archivo de las universidades públicas. 
Por la naturaleza de su nacimiento tardío, el AUROL ha mantenido un compromiso constante con el rescate de los documentos, consecuencia natural de la acumulación de éstos -empieza funciones con más de 60 años de atraso- y debido a situaciones especiales que agravan el escenario de una deficiente gestión documental, por ejemplo, unidades que habían perdido o eliminado documentos o aquéllas que, por el contrario, conservaban todo, incluidos documentos sin valor secundario.

El AUROL conserva y protege la memoria institucional; además, como instancia coordinadora tiene el propósito de regular los documentos transferidos, la mayoría de los cuales no tiene una adecuada organización, por lo que la tarea de organizar la documentación recibida generó el efecto "bola de nieve" -crecimiento exponencial-, agravado por la labor diaria, en la que impera la ausencia de políticas y procedimientos para el adecuado tratamiento y protección de la producción documental.

No obstante el panorama caótico, el AUROL, teniendo clara la meta de los archivos universitarios -crear una cultura de información archivística, que integre la gestión documental, la gestión de la información y la gestión del conocimiento en el ámbito institucional-, se propuso normalizar la producción, tratamiento y conservación del patrimonio documental institucional en todos los niveles y, aún más, frente al avance de las TIC, la globalización y la sociedad del conocimiento.

Como regulador de los diferentes archivos de la Universidad de Costa Rica, el AUROL reordena ideas y busca perspectivas de solución para enfrentar la desorganización de los documentos, faena que le llevó a reorganizar su estructura interna a través de un nuevo apartado: la sección de normalización.

Ante la necesidad de enfrentar los retos que implicaba el documento electrónico, en 2010 el AUROL inicia varios proyectos cuya finalidad era que éstos estuvieran bien organizados. Si bien el tema de las comunicaciones electrónicas no es nuevo, hasta entonces no se había tratado de forma integral.

Para contextualizar, es importante indicar que el Estado costarricense cuenta con una legislación para implementar las tecnologías de la información y comunicación bajo principios racionales de eficiencia en el uso de recursos y efectividad en su aplicación, con el objetivo de garantizar 
la eficiencia y la transparencia administrativas, así como de brindar mejores servicios a los ciudadanos, a saber:

- Ley General de la Administración Pública № 6227 de 1978.

- Ley de Protección al Ciudadano del Exceso de Requisitos y Trámites Administrativos No 8220 de 2002.

- Ley de Certificados, Firmas Digitales y Documentos Electrónicos $\mathrm{N}^{\circ} 8454$ de 2005.

- Decreto Ejecutivo de Creación de la Comisión Intersectorial de Gobierno Digital No 35139-mP-mideplan. Publicado en el Diario Oficial La Gaceta, No 67, el 6 de abril de 2009.

- Decreto Ejecutivo N³5776. Promoción del Modelo de Interoperabilidad en el Sector Público. Publicado en el Diario Oficial La Gaceta, No 41, el 1 de marzo de 2010.

- Ley Protección de la Persona Frente al Tratamiento de sus Datos Personales No 8968. Publicada en el Diario Oficial La Gaceta, No 170 , el 5 de septiembre de 2011.

- Política de Formatos Oficiales de los Documentos Electrónicos Firmados Digitalmente. Dirección de Certificadores de Firma Digital, Ministerio de Ciencia y Tecnología, 2012.

- Directriz No 067-MicitT-H-MEIC. Masificación de la implementación y uso de la Firma Digital en el Sector Público Costarricense. Publicada en el Diario Oficial La Gaceta, No 79, el 25 abril de 2014.*

La normatividad es un marco de trabajo para desarrollar sistemas informáticos que permite a las instituciones públicas agilizar sus servicios, procurando mejores niveles de eficiencia operativa, con garantía de autenticidad e integridad, a través de una herramienta de identificación confiable y segura como es la firma digital -dejando de lado el soporte papel.

Sin embargo, a pesar de dicha normativa que apunta hacia la modernización del aparato estatal, la realidad es que son casi nulos los servicios que se le brindan a los ciudadanos por estas vías o que cuentan

* Se respetó la ortografía original. 
con las garantías citadas. Los sistemas que se implementan continúan siendo islas de información que no tienen capacidad de cruzar datos entre sí; no cumplen con su propósito de simplificar trámites; lo peor, desde el punto de vista archivístico, es que no cuentan con una adecuada gestión de los documentos producidos, ni tienen mecanismos que aseguren la integridad, autenticidad, confiabilidad y preservación de la información, por lo que, en el mejor de los casos, ésta termina imprimiéndose en soporte papel para ser conservada.

Como acertadamente indica la Norma Internacional ISO 30301, el éxito de las organizaciones depende, en gran medida, de la implementación y mantenimiento de un sistema de gestión, diseñado para la mejora continua de su desempeño, mediante una metodología encaminada a la consecución de las metas de la organización, para la toma de decisiones y la gestión de recursos.

\section{La gestión de documentos}

La gestión de documentos tiene como principio fundamental determinar y controlar la trazabilidad de los documentos durante su gestión, y hasta su disposición final.

Las organizaciones tienen la necesidad de conocer y gestionar la información que producen y reciben, y con ello analizar los resultados de sus servicios y productos; esto se puede lograr por medio de la gestión documental.

La normalización de la gestión documental es un aspecto importante en toda institución, pues habilita el análisis y la mejora continua de su desempeño, la toma de decisiones y la gestión de recursos, ya que posibilita la eficiencia, la rendición de cuentas, la gestión de los riesgos y la preservación a mediano y largo plazos de la memoria institucional.

La ausencia de sistemas de información que integren políticas de gestión documental y garantías de integridad se debe, en gran medida, a un desconocimiento generalizado en relación con el tema de la administración documental.

A través del tiempo, las instituciones han visto la conservación de documentos como una imposición; y a los archivos, como un gasto, pues por muchos años el propósito del archivo era, únicamente, conservar los 
documentos para fines de investigación histórica, a veces ajena al trabajo diario de la institución productora. Aunque contribuir con la memoria histórica continúa siendo un aspecto muy importante, a veces se percibe como un proceso que no genera impacto en la institución y, por tanto, se consideraba un recurso para terceros que debía mantenerse por una disposición legal arbitraria.

Esta visión cambia radicalmente cuando los archivos se perciben como documentos administrativos que sustentan la gestión. Partiendo de esta nueva visión administrativa, se crean, en primera instancia, archivos centrales y de gestión -para el control de los documentos-, cambiando el concepto de custodia de archivos al de administradores de la información, producto de sus actividades diarias y de su interacción con la comunidad y el país donde se desenvuelven.

En el ámbito de las Universidades Estatales de Costa Rica, este proceso de cambio inició con la creación de los llamados "Archivos de gestión centralizados", cuyo propósito era darle a los documentos tratamiento archivístico desde su creación, centralizando los documentos recibidos y producidos y colaborando en la gestión administrativa

Pero el catalizador del cambio -y que sigue siendo un reto para los archivistas- es la inclusión de la tecnología en las organizaciones, ya que conlleva no sólo asumir nuevos roles y responsabilidades para una adecuada gestión de los documentos electrónicos, sino garantizar su autenticidad, integridad y confiabilidad a través del tiempo.

Una adecuada gestión documental, al igual que el desempeño profesional, es determinante para la transparencia, la toma de decisiones y la preservación de la memoria institucional -mediante la aplicación de normas archivísticas de calidad.

La gestión documental es una función clave que, para que pueda percibirse, debe estar alineada con los objetivos de la institución, orientada a los procesos sobre los que se desarrollan sus estrategias e integrada -desde el mismo diseño- con los sistemas de información y gestión que dan soporte a estos procesos.

El AUROL de la Universidad de Costa Rica ha realizado acciones encaminadas al cumplimiento de lo establecido por el Reglamento del Sistema de Archivos, con el fin de lograr un adecuado funcionamiento del Sistema de 
Archivos Universitarios (Sau) mediante mecanismos de normalización. Tales acciones están alineadas con las siguientes políticas de la institución:

- 4.1.3. Fortalecerá la simplificación de los procesos académicos y de los trámites administrativos.

- 4.1.5. Fortalecerá una cultura de transparencia y rendición de cuentas de todas las actividades y del uso de los recursos.

- 4.3.1. Fortalecerá el sistema integrado de información universitaria, para coadyuvar en los procesos y en la toma de decisiones institucionales, con base en principios de seguridad, confidencialidad, universalidad y accesibilidad. ${ }^{2}$

\section{Proyecto de normalización}

Como resultado de los esfuerzos por el cumplimiento de sus funciones y de las políticas y estrategias institucionales, y debido a que el AUROL debe regular la articulación y la coordinación de los diferentes archivos de la Universidad de Costa Rica, se inicia un proyecto para implementar la norma ISO 15489 en toda la institución. El piloto se aplica en dos oficinas -la rectoría y el archivo universitario- con el fin de aprovechar la experiencia y avanzar en la aplicación de las mejores prácticas archivísticas a nivel institucional.

El objetivo del proyecto fue normalizar la gestión de documentos, desarrollando políticas y procedimientos que aseguraran su adecuado diseño, creación, conservación, recuperación y uso a lo largo del tiempo, y que a su vez contribuyeran con la ejecución de las actividades, la prestación de servicios de manera eficaz, la toma de decisiones y la asunción de responsabilidades a nivel institucional.

Para el desarrollo del proyecto se utilizó la metodología propuesta por la norma 15489 para diseñar e implementar el sistema de gestión de documentos y establecer las actividades de evaluación, medición y aprendizaje encaminadas a la mejora continua.

Se realizó un análisis y recopilación de información -mediante una investigación preliminar- que permitió identificar los contextos interno,

2 Políticas de la Universidad de Costa Rica para los años 2010-2014. 
externo, jurídico, funcional, administrativo y documental del AUROL y delimitar su papel dentro y fuera de la institución, así como una visión de las fortalezas, oportunidades, debilidades y amenazas a las que se pueda enfrentar.

Simultáneamente, se realizó un diagnóstico de la situación actual de la gestión de los documentos en la Universidad de Costa Rica, con el cual se identificaron los elementos que afectan positiva o negativamente la gestión documental, detectando posibles áreas de riesgo.

El análisis de las actividades de la organización se realizó mediante el enfoque basado en procesos; se identificaron los Macroprocesos: docencia, investigación, acción social, vida estudiantil, administración, gestión estratégica.

En primera instancia, se realizó el análisis de las actividades y procesos del AUROL y de rectoría por medio de un mapeo, permitiendo visualizar los puntos en los que se producen o reciben los documentos como producto de la actividad de cada oficina.

Se realizó un análisis de los requisitos técnico administrativos, legales y tecnológicos y de las necesidades de los usuarios, para el diseño, producción, utilización, mantenimiento y evaluación de los documentos de la rectoría y del AUROL.

En cuanto a la evaluación de los sistemas existentes, la norma ISO 15489 posee un apartado específico. En este ámbito de trabajo se analizó el sistema de información llamado GCI (utilizado por algunas instancias universitarias), con el fin de analizar el cumplimiento de los requisitos identificados en la etapa anterior. Se analizaron criterios estratégicos, funcionales y técnicos, tomando en cuenta los aspectos que contempla la Ley General de Control Interno $\mathrm{N}^{\circ}$ 8292: seguridad, confiabilidad, adaptabilidad, normalización de los procesos de gestión de documentos, control, generación de reportes, acceso y uso de la información.

El GCI y el conocimiento acumulado en su entorno sirvieron de base para el diseño del nuevo sistema electrónico de gestión documental institucional llamado SisDoc, cuyo fin es mejorar áreas deficientes e implementar nuevas funcionalidades y características de seguridad, integridad y autenticidad acordes con la normativa y necesidades vigentes, de manera que se satisfagan las necesidades identificadas para la gestión de los documentos. Cabe destacar que como parte del proceso se identificaron las políticas, 
procedimientos, instrumentos y mecanismos que hace falta diseñar e implementar para remediar los puntos débiles detectados, no solamente para el AUROL, sino a nivel institucional.

Como resultado de ese ejercicio -en conjunto con la rectoría- se establece el plan de implementación del SisDoc, con el fin de mejorar y garantizar una adecuada gestión documental automatizada y posibilitar la preservación de la memoria histórica en soporte digital a través de un sistema docucéntrico.

El SisDoc permite crear y enviar documentos con las características y requisitos identificados, clasificar y describir los documentos que se generan y se reciben; recuperar la información, realizar transferencias, seleccionar, valorar y eliminar documentos, y controlar el servicio de préstamo. Actualmente el AUROL se encuentra en etapa de implementación del sistema en otras instancias universitarias.

Con el fin de normalizar la producción, uso y conservación de los documentos, se han elaborado otros instrumentos que son subproductos del análisis, entre los que figuran el Procedimiento para la Gestión de las Actas y los Expedientes de Sesiones de los Órganos Colegiados de la Universidad de Costa Rica - difundidos a la comunidad universitaria a través de capacitaciones en conjunto con la Oficina Jurídica, la Oficina de Contraloría Universitaria, el Consejo Universitario y la Oficina de Recursos Humanos- y la Directriz AUROL-2: elaboración de cartas, circulares y memorandos oficiales, tomando en cuenta los requisitos identificados y los parámetros que establece el manual de identidad visual de la Universidad de Costa Rica.

Asimismo -con el apoyo de estudiantes de ingeniería industrial-, se analizaron procesos de toda la institución para generar un mapa de series, el cuadro de clasificación basado en procesos y actualizar las tablas de plazos generales.

Se trabajaron las políticas por implementar en la institución mediante asesores expertos en conjunto con el Centro de Informática y la Sección de Archivística. El resultado de este esfuerzo fue la Política de Preservación de Documentos Electrónicos (PPDE), que atiende las necesidades actuales de la universidad -que no resultan muy distintas de los retos que enfrentan otras instituciones dentro y fuera del país- en el ámbito de documentos electrónicos. 
La PPDE es un instrumento moderno, con una visión integral alineada a los marcos mundiales de mejores prácticas, que se convertirá en un modelo de consulta para los interesados en enfrentar los retos de la preservación del documento en soporte digital.

El trabajo realizado en el proyecto de normalización AUROL consideró la evaluación del ambiente, el análisis de la situación, la delimitación del contexto relativo a obligaciones y voluntad, labor orientada hacia el compromiso de la institución de disponer de un archivo digital, considerando la previsión de las dimensiones, estimación de ingreso, crecimiento anual, cantidad de usuarios, los niveles de control y restricciones de acceso.

También se evaluaron riesgos para definir una estrategia corporativa de preservación digital y una comisión directora del nuevo archivo digital con la participación del personal implicado.

Con la aprobación de la PPDE y la conformación de la comisión interdisciplinaria se iniciarán las investigaciones necesarias para implementar un archivo digital, que permita el almacenamiento, la clasificación, el acceso seguro y, eventualmente, la migración ordenada y eficiente de la información y los documentos resguardados conforme con los nuevos formatos y soportes electrónicos con visión al futuro.

Estas acciones son solamente un ejemplo del beneficio de una correcta y ordenada gestión documental, trabajo que las instituciones, sobre todo las de educación superior, deben considerar como una herramienta que permite la adecuada administración, de acuerdo con las exigencias actuales.

Las universidades deben identificar el valor de su información y de la normativa que les afecta, vinculando los sistemas con el contexto normativo y documental, así como mantener un control sistemático de los vínculos de los documentos en soporte electrónico con el software que garantice su uso y acceso, mediante la revisión periódica de la obsolescencia de los sistemas y los mecanismos procedimentales para la sustitución o exportación de los datos. No obstante, nada de esto sería posible sin considerar primero a la gestión de la producción documental, que es el primer eslabón de la cadena evolutiva de los sistemas de información, cuyo primer paso consiste en cambiar la concepción de un proceso operativo a un proceso estratégico. 


\section{Conclusiones}

La educación es fundamental para la sociedad pues produce y distribuye el recurso esencial de la evolución social y económica de los pueblos: "el conocimiento".

Los archivos son entes posibilitadores del acceso oportuno, exacto y veraz al conocimiento producido o recibido por la institución.

La labor del archivo es muy importante en toda organización, no sólo para interpretarse a través del tiempo, sino para garantizar el acceso a la información, la transparencia de la gestión administrativa y sustentar la toma de decisiones, a través de una gestión eficiente.

Los archivos tienen todo un reto, no sólo por la gestión de los documentos y la cultura organizacional en las instituciones, sino porque tienen la responsabilidad de enfrentar y administrar los retos que las transformaciones sociales y tecnológicas imponen sobre la salvaguarda del patrimonio documental, la transparencia de la gestión pública y la mejora continua de la eficiencia en los procesos administrativos.

La archivística ha evolucionado de acuerdo con las necesidades de información en el contexto de la sociedad en que se desenvuelve; sin embargo, no al ritmo necesario para beneficiarse plenamente de esos cambios. Actualmente, en plena era digital, la archivística ha tenido que replantearse para dar gestión a las nuevas formas de crear y custodiar los documentos.

Uno de los retos por enfrentar para los documentos es el tiempo entre su producción en soporte electrónico y la definición de métodos de preservación, considerando que la mayoría de las soluciones tiene una caducidad menor que el soporte papel. Esta característica obliga a plantear paradigmas y prevenir los posibles riesgos que obligan a llevar a cabo un análisis con una perspectiva diferente para la conservación de los objetos digitales. Esta nueva administración de procesos hace vital -más que nuncala gestión de documentos en el movimiento de maduración para convertirse en un proceso estratégico.

Los documentos contienen información que define lo que somos y las características de la sociedad. Este patrimonio documental es el constructo de la memoria colectiva. Como archivistas, es nuestro deber y orgullo 
brindar la información para la adecuada toma de decisiones y colaborar con la transparencia institucional y la rendición de cuentas. Los documentos son, y serán, la fuente que contará a nuestros sucesores quiénes fuimos y de dónde venimos. Cabe destacar en este punto la función del acervo documental; es una herramienta para comprender la historia, pues contiene la información que define hacia dónde iremos.

\section{Bibliografía}

AENOR, Norma española UNE-ISO 15489. Información y documentación. Gestión de documentos, 2006.

AENOR, Norma española UNE-ISO 30300. Información y documentación. Sistema de gestión para los documentos. Fundamentos y vocabulario, 2011.

AENOR, Norma española UNE-ISO 30301. Información y documentación. Sistemas de gestión para los documentos: Requisitos, 2011.

Bermúdez Muñoz, María Teresa, La gestión de documentos. Visión panorámica, en: http://www.archivonacional.go.cr/memorias/1995/01.pdf

Chacón Arias, Virginia, "El ayer y el hoy del desarrollo archivístico costarricense", en Revista del Archivo Nacional de Costa Rica, año LXV, San José, 2001, pp. 27-47.

"Decreto Ejecutivo $\mathrm{N}^{\circ}$ 35776. Promoción del Modelo de Interoperabilidad en el sector Público", en Diario Oficial La Gaceta, núm. 41, 1 de marzo de 2010.

"Directriz No 067-MICITT-H-MEIC. Masificación de la implementación y el uso de la firma digital en el sector público costarricense", en Diario Oficial La Gaceta, núm. 79, 25 de abril de 2014.

"Ley de Protección de la persona frente al tratamiento de sus datos personales No 8968", en Diario Oficial La Gaceta, núm. 170, 5 de septiembre de 2011. "Política de Formatos Oficiales de los Documentos Electrónicos Firmados Digitalmente", en Dirección de Certificadores de Firma Digital, Ministerio de Ciencia, Tecnología y Comunicaciones, 2012.

Rivas Fernández, José Bernal, "La formación de archivistas en Costa Rica". Memoria del XX Congreso Archivistico Nacional. Archivo Nacional de Costa Rica, 2008. 
Serra Serra, Jordi, "La firma electrónica y el archivo digital", en Primeres Jornades de Signatura Electrònica. Agència Catalana de Certificació (CATCert), Barcelona, 10-11 de junio de 2004.

Gestión y conservación de los documentos electrónicos desde la perspectiva archivística: un nuevo escenario de actuación, en I Congreso sobre gestión y conservación del documento electrónico, Universitat Jaume I (Castelló), 2007.

Universidad de Costa Rica, "Políticas de la Universidad de Costa Rica para los años 2010-2014”, en La Gaceta Universitaria, 40, 2008. 